\title{
Emptiness of the left iliac fossa: a new clinical sign of sigmoid volvulus
}

\author{
V Raveenthiran
}

\begin{abstract}
Background-Diagnostic difficulties are common in sigmoid volvulus. This diagnostic delay contributes to high morbidity and mortality.

Aim-This paper recognises visible or palpable emptiness of the left iliac fossa as a new clinical sign of sigmoid volvulus. Predictive accuracy of the sign was assessed in this study.

Methods-519 patients with acute abdomen of non-traumatic origin were prospectively studied.

Results-The new sign was present in 24 out of 86 patients $(28 \%)$ with sigmoid volvulus. It was absent in all those who did not have sigmoid volvulus. The positive predictive value of the sign was $100 \%$.

Conclusion-Emptiness of the left iliac fossa is a valuable diagnostic adjunct. Positive sign is pathognomonic of sigmoid volvulus. Nevertheless, a negative sign warrants further investigations. (Postgrad Med F 2000;76:638-641)
\end{abstract}

Department of Surgery, Veer Surendra Sai Medical College, Sambalpur, Orissa, India

Correspondence to: Dr V Raveenthiran, 190/1 Eighth Street, Sayee Nagar, Chennai 600 092, India (email:

venkatsankar@yahoo.com)

Submitted 25 January 2000 Accepted 9 May 2000

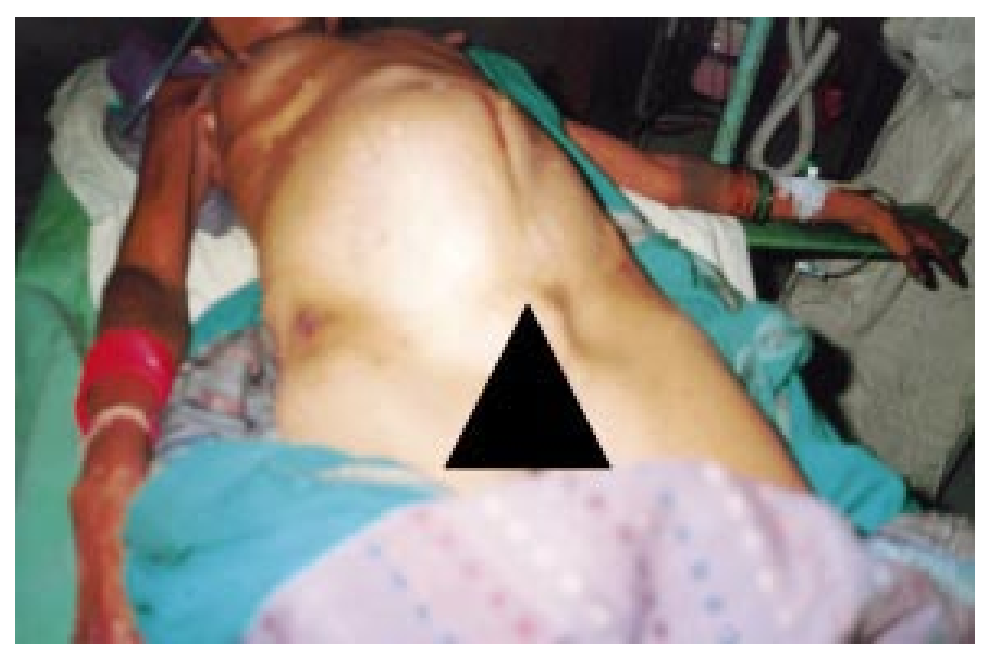

Figure 1 Clinical photograph showing emptiness of the left iliac fossa in a case of sigmoid volvulus (reproduced with the patient's permission). diagnose the condition. Diagnostic difficulties, however, are not uncommon. A recent report from Finland, where the disease is endemic, confirms the problem of poor diagnostic accuracy. ${ }^{2}$ This paper describes a new physical sign that complements the diagnosis of sigmoid volvulus.

\section{The Sign}

Abdominal distension in sigmoid volvulus exhibits a peculiar asymmetry. There is often a relative emptiness in the left iliac fossa (fig 1 ). It is better appreciated from the foot end of the bed while the patient lies supine with stretched legs. Oblique lighting enhances the perception of emptiness. Alternatively, palpation by trained hands will quickly reveal a relative emptiness of the left iliac fossa compared with that of the right side.

\section{Patients and methods}

From March 1994 to November 1996, 519 patients were admitted to the author's department with acute abdominal pain of nontraumatic origin. Patients with a history of blunt or penetrating injury to the abdomen were excluded from the study. Diagnosis was uncertain in 36 patients for two reasons: some of them died soon after arrival and others became well in the absence of a confirmed clinical diagnosis. Mesenteric lymphadenitis, amoebiasis, enterocolitis, dyspepsia, and salpingitis were frequently the clinical diagnosis in the latter group. The remaining 483 patients were prospectively studied. Final diagnosis was made in these patients by either surgical exploration or appropriate investigations such as radiographs and biochemical estimations.

\section{Results}

There were 86 patients with sigmoid volvulus. The remaining 397 patients belonged to various diagnostic categories: perforated peptic ulcer (113), acute appendicitis (96), obstructed hernia (51), adhesive bowel obstruction (39), imperforate anus (22), ureteric calculi (18), paralytic ileus (11), acute pancreatitis (7), colorectal carcinoma (6), ileosigmoid knotting (6), jejunoileal volvulus (6), ileal stricture due to tuberculosis (5), caecal volvulus (2), and miscellaneous (15).

The mean age of sigmoid volvulus patients was 49 years (range 19-75) and male to female ratio was 1:1. The mean age of the "nonsigmoid volvulus" group was 27 years (range $0-82$ ) and sex ratio was $2: 1$. The sign was 
Box 1: Radiographic signs of sigmoid volvulus

- Bent inner tube appearance

- Omega sign (inverted U shaped sigmoid colon)

- Coffee bean sign

- Frimann-Dahl's sign (three linear shadows converging to the left side)

- Y sign

- Horseshoe sign

- Loss of colonic haustrations

- Left flank overlap sign (overlap of sigmoid and descending colon)

- Liver overlap sign

- Apex of sigmoid colon loop above the level of 10 th thoracic vertebra

- Apex of sigmoid colon loop under the left dome of diaphragm

- Absence of gas shadow in the left iliac fossa

- Air fluid ratio greater than 2:1

- Thickening of colonic wall

- Northern exposure sign ${ }^{9}$
Box 2: Differential diagnosis of sigmoid volvulus

- Colorectal carcinoma causing obstruction

- Toxic megacolon

- Colorectal strictures

- Hirschsprung's disease

- Caecal volvulus

- Paralytic ileus

- Ileosigmoid knotting

- Ogilvie's disease (colonic pseudo-obstruction)

- Acquired megacolon

- Giant colonic diverticulum

Box 3: Features of sigmoid volvulus in barium enema

- Bird's beak appearance

- Ace of spade sign

- Spiral mucosal pattern (corkscrew sign)

- Redundant atonic sigmoid colon (in recurrent volvulus)

Clinical cues are often supplemented with plain and/or contrast radiography. Several signs of sigmoid volvulus have been described in plain radiographs (box 1) ${ }^{7-9}$ However, many of them are non-specific.

Hence, sigmoid volvulus may be mistaken for a variety of conditions or vice versa (box 2). Plain radiographs are diagnostic of sigmoid volvulus in only $30 \%$ to $40 \%$ cases. ${ }^{8}{ }^{10}$ Some authors, ${ }^{11}$ however, claim a diagnostic accuracy of $60 \%$ to $80 \%$ in plain films. In doubtful cases a barium enema is recommended both as a diagnostic and a therapeutic method (box 3). Several large series agree that barium enema is diagnostic in only $20 \%$ to $30 \%$ of cases. ${ }^{10}{ }^{11}$ Moreover, instillation of barium in a case of undiagnosed acute abdomen carries the risk of barium peritonitis. ${ }^{8}$ Diagnostic accuracy of plain and contrast radiography in children with sigmoid volvulus is $29 \%$ and $61 \%$ respectively. ${ }^{12}$ Computed tomographic features of sigmoid volvulus such as whirlpool sign and kissing beak appearance have recently been described. ${ }^{13}$ Although endoscopy has gained popularity as the therapeutic method of choice, it is generally considered as a poor diagnostic tool. Thus, a small subset of patients with sigmoid volvulus is operated upon without a definitive or with an erroneous preoperative diagnosis. The sign described in this paper may be useful in such cases.

Although surgical exploration itself is an accepted mode of diagnosing acute abdomen, accurate preoperative diagnosis will reduce the morbidity and mortality. For example, an ill chosen incision based on an erroneous preoperative diagnosis will interfere with the proper fashioning of a colostomy, should it be necessary in the course of surgery. 
Normal

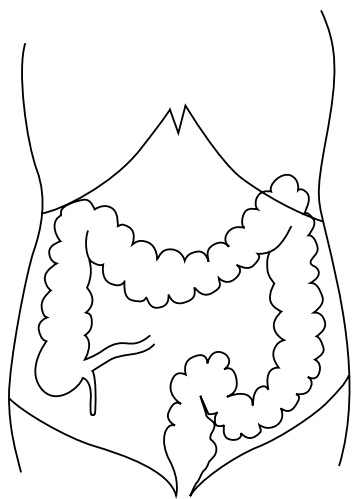

Volvulus

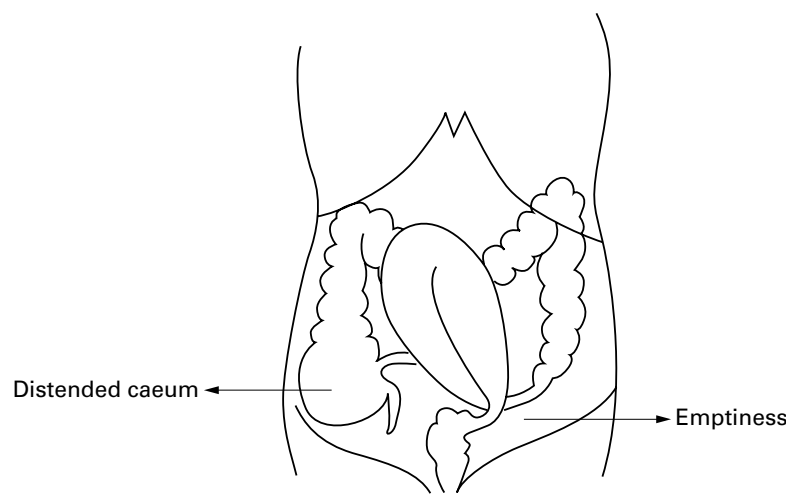

Figure 2 Diagrammatic representation of the probable mechanism of the new sign. Normal anatomy is also shown for comparison.

The exact mechanism of the new sign is not known (fig 2). Redundancy of mesosigmoid is a predisposing factor of volvulus. ${ }^{1}$ Due to its long mesentery, the twisted loop of sigmoid colon rises out of the pelvis and tends to occupy the right side of the abdomen (figs 2 and 3). Because of this migration, emptiness is created in the left iliac fossa. Operating experience suggests that the migratory segment of sigmoid sweeps the coils of small intestine to the right side. Thus, they are prevented from occupying the left iliac fossa. Moreover, when the ileocaecal valve is competent, closed loop obstruction occurs not only in the twisted loop of the sigmoid but also in the colon proximal to it. As in any other colonic obstruction, the caecum bears the brunt and distends maximally. The caecal distension on the right side probably contributes to the relative emptiness of the left iliac fossa by exaggerating the asymmetry of abdominal distension (fig 2).

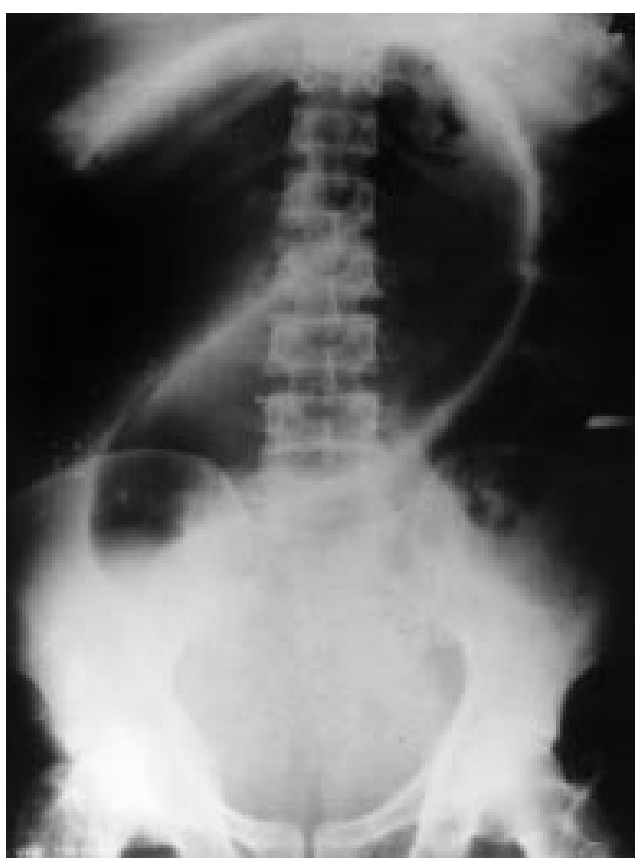

Figure 3 Plain radiograph of patient with sigmoid volvulus explaining why the sign is present in some cases. Twisted loop of sigmoid has migrated to the right upper quadrant leaving the left iliac fossa empty. The new sign was present in this patient.
Like any other physical sign, limitations and fallacies of this new sign must be understood to appreciate its usefulness. Predictive value of positive sign is $100 \%$, but its sensitivity is only $28 \%$. Thus, when the sign is positive it is pathognomonic of sigmoid volvulus and further investigations are probably unnecessary. Absence of the sign, however, does not predict anything. Asymmetric distension of abdomen is a theoretical possibility in adhesive obstruction. Nevertheless, it was not encountered in any of the 39 cases in this series. Other types of volvulus such as caecal volvulus may possibly produce asymmetric distension, but not emptiness of the left iliac fossa. Reason for the absence of the sign in some cases is not known. It depends probably on the migratory nature of the twisted colon. In some cases, the loop migrates to the right side of abdomen (fig 3)

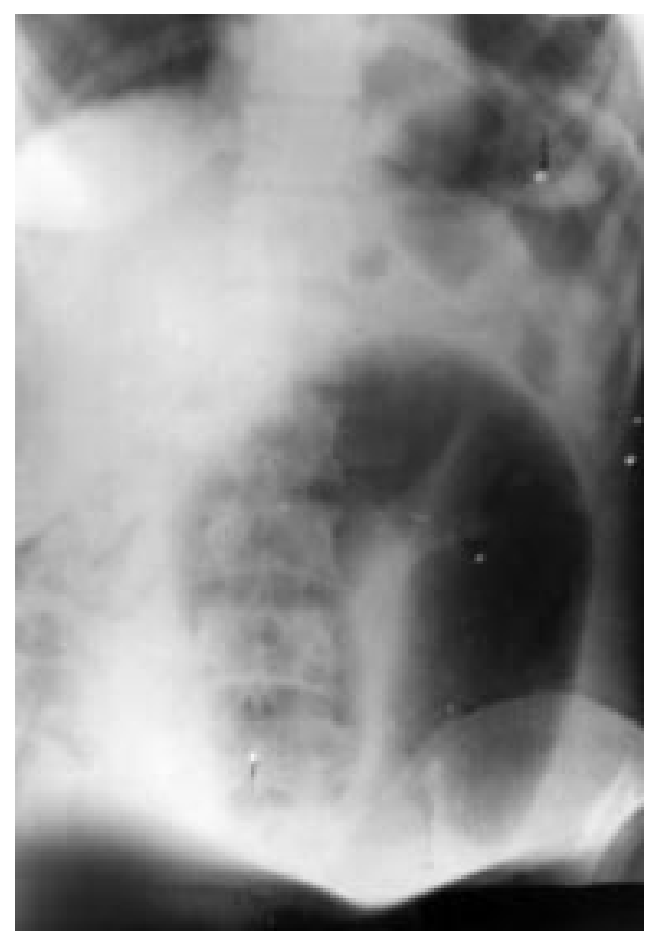

Figure 4 Plain radiograph of patient with sigmoid volvulus explaining why the sign is absent in some cases. Twisted loop remains in the left flank. The new sign was absent in this case. 
while in others it remains in the left flank (fig 4). Conceivably, the sign is positive in the former and negative in the latter.

In conclusion, emptiness of the left iliac fossa is recommended as a valuable diagnostic adjunct. The sign is highly specific but poorly sensitive of sigmoid volvulus. Negative sign warrants further investigations and a positive sign is pathognomonic of sigmoid volvulus.

1 Shepherd JJ. The epidemiology and clinical presentation of sigmoid volvulus. Br F Surg 1969;56:353-9.

Hiltunen KM, Syrja H, Matikainen M. Colonic volvulus: diagnosis and results of treatment in 82 patients. Eur F Surg 992;158:607-11

3 Ballantyne GH. Review of sigmoid volvulus. Dis Colon Rectum 1982;25:494-501

4 String ST, DeCosse JJ. Sigmoid volvulus: an examination of the mortality. Am F Surg 1971;121:293-7.
5 Hinshaw DB, Carter R. Surgical management of acute volvulus of the sigmoid colon: a study of 55 cases. Ann Surg 1957; 146:52-60.

6 Clain A. Hamilton Bailey's demonstration of physical signs in clinical surgery. 17th Ed. Bristol: John Wright, 1986: 314-15.

7 Burrell HC, Baker DM, Wardrop P, et al. Significant plain film findings in sigmoid volvulus. Clin Radiol 1994;49:31719.

8 Field S. The acute abdomen. In: Sutton D, ed. A textbook of radiology and imaging. Vol 2 . 5 th Ed. London: Churchill Livradiology and imaging. Vol

9 Javors BR, Baker SR, Miller JA. The northern exposure

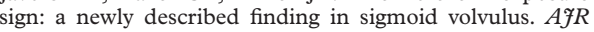
1999;173:571-4.

10 Ballantyne GH, Brandner MD, Beart RW, et al. Volvulus of the colon: incidence and mortality. Ann Surg 1985;202:8392.

11 Arnold GJ, Nance FC. Volvulus of the sigmoid colon. Ann Surg 1973;177:527-34.

12 Smith SD, Golladay ES, Wagner C, et al. Sigmoid volvulus in childhood. South Med f 1990;83:778-81.

13 Catalano O. Computed tomographic appearance of sigmoid volvulus. Abdom Imaging 1996;21:314-17. 